\title{
Comparison of Clinical Characteristics Between Obese and Non-Obese Patients with Nonalcoholic Fatty Liver Disease (NAFLD)
}

\author{
Yifang Li \\ Yanzhen Chen \\ Xing Tian \\ Shanshan Zhang \\ Jian Jiao
}

Department of Gastroenterolgy \& Hepatology, China-Japan Union Hospital, jilin University, Changchun, I30033, People's Republic of China
Correspondence: Jian Jiao

Department of Gastroenterology \& Hepatology, China-Japan Union Hospital, jilin University, Changchun, People's Republic of China

Tel $+86 \quad 13756009567$

Fax +86043 I-84995850

Email jjian@jlu.edu.cn
Objective: Non-alcoholic fatty liver disease (NAFLD), previously thought to predominantly affect obese individuals, has also been shown to occur in subjects who have a relatively normal body mass index (BMI). Due to the normal BMI, non-obese NAFLD are easily to be ignored and eventually lead to potential liver injuries.

Methods: A population-based cross-sectional study was conducted on 1608 cases with normal serum alanine aminotransferase (ALT) levels who were divided into an obese group (BMI $\geq 25 \mathrm{~kg} / \mathrm{m}^{2}$ ) and a non-obese group (BMI $<25 \mathrm{~kg} / \mathrm{m}^{2}$ ). NAFLD was diagnosed by ultrasound and Fibro Scan examination. Non-obese populations were divided into NAFLD group (CAP $\geq 240 \mathrm{db} / \mathrm{m}$ ) and non-NAFLD group (CAP $<240 \mathrm{db} / \mathrm{m})$. The incidence of NAFLD in the obese and non-obese populations and constituent ratios of genders, age, and serum levels of triglycerides (TG), cholesterol (CHOL), and blood glucose were compared. Risk factors of NAFLD in non-obese people were analyzed by multivariate logistics regression.

Results: The occurrence of NAFLD was higher in the obese group than in the non-obese group, regardless of gender $(\mathrm{P}<0.001)$. In the non-obese group, the occurrence of NAFLD in female patients was lower than that in male $(\mathrm{P}=0.001)$. The occurrence of NAFLD increased with age, with 50-59 years being the peak age of incidence in both male and female. The peak age of NAFLD occurrence in non-obese male patients was more delayed than that in obese male patients. $\mathrm{BMI}(\mathrm{OR}=1.311, \mathrm{P}=0.000)$ and $\mathrm{TG}(\mathrm{OR}=2.545, \mathrm{P}=0.000)$ were risk factors for NAFLD in the non-obese population.

Conclusion: Compared with obese population, the incidence of NAFLD in non-obese population was relatively low and more frequently in male than in female, the peak age of NAFLD occurrence in non-obese male patients was also delayed. BMI and TG should still be controlled to avoid the occurrence of NAFLD although the BMI of such patients is normal.

Keywords: obese, non-obese, non-alcoholic liver disease, gender, characteristic

\section{Introduction}

Non-alcoholic fatty liver disease (NAFLD), previously thought to predominantly affect obese individuals, ${ }^{1}$ has also been shown to occur in subjects who have a relatively normal body mass index (BMI), a condition referred to as non-obese or lean NAFLD, ${ }^{2}$ and is occult and therefore easily ignored in clinical examinations. ${ }^{3}$ Studies have reported that the incidence rate of NAFLD in nonobese population is $7-20 \%$ in the West and $5-26 \%$ in the East, ${ }^{4}$ however, current studies on NAFLD are mainly focused on obese patients, and there is 
a lack of research on non-obese patients, the clinical characters and the risk factors of non-obese NAFLD remain poorly defined, sexual dimorphism, advanced age or postmenopausal status, and serum levels of lipid profile and blood glucose levels may be involved. ${ }^{5}$ Considering the body figures are different between the Eastern and the western, this paper takes BMI $\geq 25 \mathrm{~kg} /$ $\mathrm{m}^{2}$ as the definition of obesity according to the recommendation of World Health Organization for the Eastern, ${ }^{6}$ the clinical characteristics between obese and non-obese patients with NAFLD were compared and the difference in the risk factors for NAFLD between nonobese male and female patients were also analyzed, the aim is to provide better understandings for non-obese NAFLD.

\section{Materials and Methods}

\section{Patients and Laboratory Assessment}

Overall, 1608 cases with normal serum alanine aminotransferase (ALT) levels undergoing routine physical examination in the Physical Examination Center of the China-Japan Union Hospital (Jilin University, China) were screened for NAFLD by abdominal ultrasound and Fibro Scan examination. The main characteristics of 1608 cases undergoing routine physical examination are shown in Table 1.

Histories of alcohol consumption and medications used within the last three months were investigated. Serum antibody levels of hepatitis virus A, B, C, and E were assessed. Alcohol consumption, virus infection, autoimmune liver disease, coronary artery disease (CAD), acute inflammatory conditions, acute and chronic liver diseases, pregnancy, organ failure, rheumatological disorders, hypothyroidism, malignancy and currently undergoing therapy were the exclusion criteria. Fatty liver was diagnosed by ultrasonographic examination indicating fatty infiltration in the liver and controlled attenuation parameter $\geq 240 \mathrm{db} / \mathrm{m}$. They were evaluated and reported by the same physician to avoid bias. The detailed diagnostic criteria were in accordance with the guidelines for NAFLD management formulated by the Chinese National Workshop on Fatty Liver Disease in $2010 .^{7}$ The research was conducted ethically in accordance with the World Medical Association Declaration of Helsinki and was approved by the Ethics Committee of China-Japan Union Hospital, Jilin University
Table I Main Characteristics of I608 Cases Undergoing Routine Physical Examination

\begin{tabular}{|l|l|l|l|}
\hline $\begin{array}{l}\text { Clinical } \\
\text { Indicators }\end{array}$ & $\begin{array}{l}\text { Non-Obese } \\
(\mathbf{n}=\mathbf{9 7 4})\end{array}$ & $\begin{array}{l}\text { Obese } \\
(\mathbf{n}=634)\end{array}$ & P value \\
\hline Age (year) & $50.39 \pm 1 \mathrm{I} .57$ & $47.2 \mathrm{I} \pm 10.86$ & 0.01 \\
Sex (male/female) & $59 / 42$ & $297 / 98$ & 0.03 \\
BMI (kg/m²) & $23.61 \pm 1.36$ & $28.53 \pm 2.64$ & 0.01 \\
E (kPa) & $6.67 \pm 1.65$ & $7.96 \pm 4.13$ & 0.00 \\
CAP (db/m) & $263.21 \pm 21.49$ & $267.35 \pm 24.94$ & 0.00 \\
AST (IU/L) & $28.65 \pm 12.27$ & $28.85 \pm 12.3$ & 0.30 \\
ALT (IU/L) & $37.06 \pm 28.18$ & $36.80 \pm 24.45$ & 0.01 \\
ALP (IU/L) & $90.40 \pm 22.96$ & $89.15 \pm 23.22$ & 0.20 \\
GGT (IU/L) & $46.28 \pm 27.52$ & $52.32 \pm 61.52$ & 0.03 \\
ALB (g/L) & $46.84 \pm 3.31$ & $46.51 \pm 2.55$ & 0.50 \\
TG (mmol/L) & $2.20 \pm 1.83$ & $2.66 \pm 2.52$ & 0.01 \\
CHOL (mmol/L) & $5.19 \pm 0.85$ & $5.35 \pm 1.06$ & 0.02 \\
HDL-C (mmol/L) & $1.14 \pm 0.24$ & $1.12 \pm 0.24$ & 0.03 \\
LDL-C (mmol/L) & $3.47 \pm 0.70$ & $3.59 \pm 0.83$ & 0.02 \\
Blood glucose & $5.79 \pm 1.35$ & $6.25 \pm 2.14$ & 0.04 \\
(mmol/L) & & & \\
\hline
\end{tabular}

Note: All measurement data are expressed as mean \pm standard deviation.

(2016ks009). Participation was voluntary and all respondents completed informed consent.

Fasting venous blood was taken in the morning (fasting for more than 8 hours and no high-fat diet one day before blood collection) for testing. Triglyceride (TG), total cholesterol (CHOL), low-density lipoprotein cholesterol (LDL-C), high-density lipoprotein cholesterol (HDL-C), alanine transaminase (ALT), aspartate aminotransferase (AST), Alkaline phosphatase (ALP), Gamma-glutamyl transferase (GGT), Albumin (ALB) and fasting blood glucose levels were detected by HITACHI-7180 automatic biochemical analyzer.

\section{Study Design}

This is a population-based cross-sectional study. According to the World Health Organization standard for Asian populations ${ }^{6}$ based on the risk of developing type 2 diabetes and cardiovascular disease, we divided the 1608 cases into an obese group (BMI $\left.\geq 25 \mathrm{~kg} / \mathrm{m}^{2}\right)$ and a nonobese group $\left(\mathrm{BMI}<25 \mathrm{~kg} / \mathrm{m}^{2}\right)$. The incidence of NAFLD in the obese and non-obese populations and its relationship with clinical indicators, including gender, age, and serum levels of triglycerides (TG), cholesterol (CHOL), and blood glucose, were compared. The schematic design is presented in Figure 1.

Non-obese populations were divided into NAFLD group $(\mathrm{CAP} \geq 240 \mathrm{db} / \mathrm{m})$ and non-NAFLD group $(\mathrm{CAP}<240 \mathrm{db} / \mathrm{m})$ according to the results of abdominal 


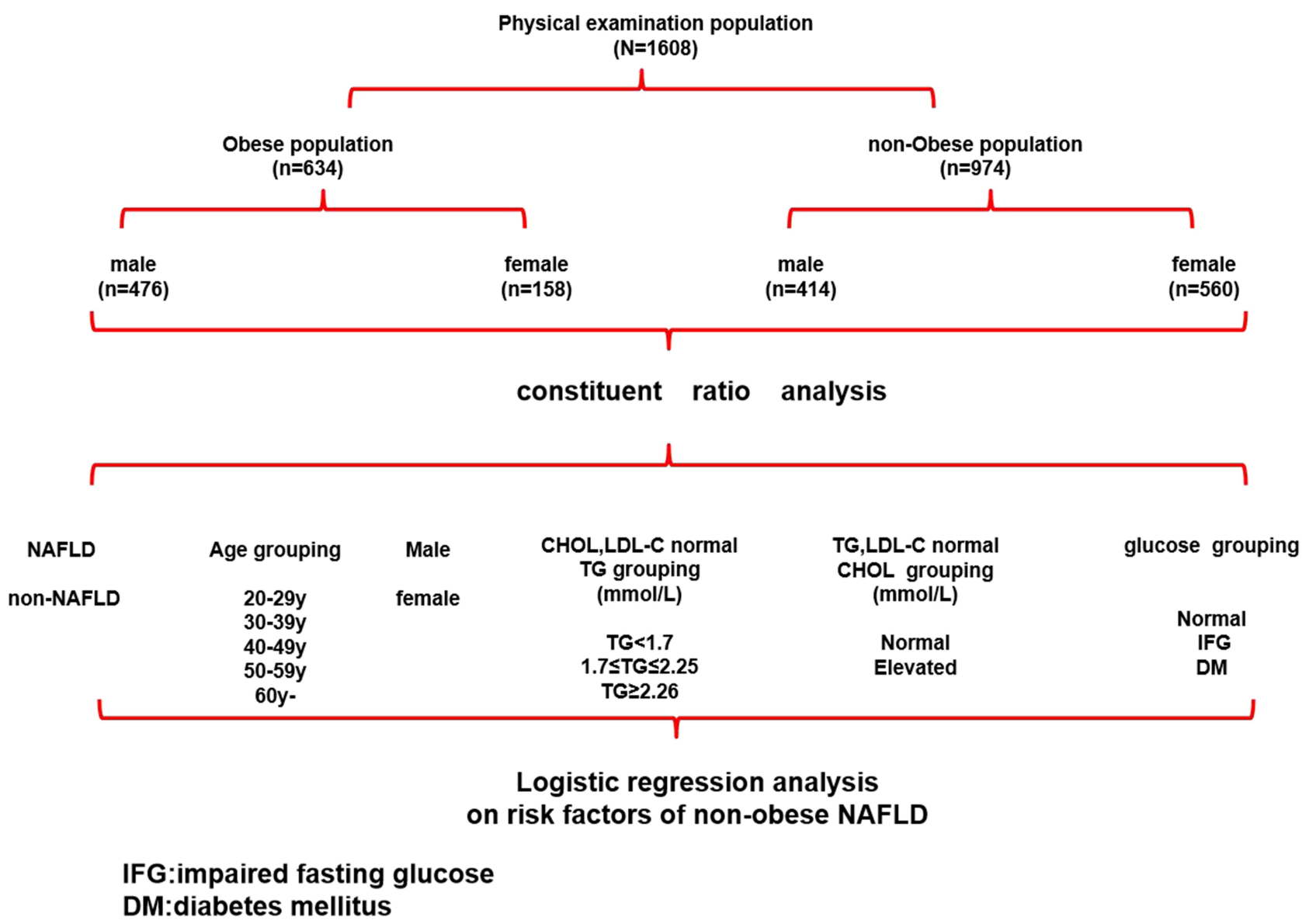

Figure I Patients and study design. 1608 cases were divided into obese group (BMI $\geqq 27.5 \mathrm{~kg} / \mathrm{m} 2)$ and non-obese group (BMI < $27.5 \mathrm{~kg} / \mathrm{m} 2)$. Constituent ratio of NAFLD in obese and non-obese population and its relationship with clinical indicators were compared. Risk factors of non-obese NALFD were analyzed.

ultrasound and Fibro Scan examination, the main characteristics of the NAFLD group and the non-NAFLD group in non-obese people are shown in Table 2.

Table 2 Main Characteristics of NAFLD Group and NonNAFLD Group in Non-Obese Population

\begin{tabular}{|l|l|l|l|}
\hline $\begin{array}{l}\text { Clinical } \\
\text { Indicators }\end{array}$ & $\begin{array}{l}\text { NAFLD } \\
(\mathbf{n}=1 \mathbf{0 I})\end{array}$ & $\begin{array}{l}\text { Non-NAFLD } \\
(\mathbf{n}=873)\end{array}$ & P value \\
\hline Age (year) & $50.13 \pm 11.83$ & $45.73 \pm 1 \mathrm{I} .26$ & 0.05 \\
Sex (male/female) & $59 / 42$ & $355 / 518$ & 0.01 \\
BMI (kg/m $\left.{ }^{2}\right)$ & $23.26 \pm 2.45$ & $21.86 \pm 2.16$ & 0.00 \\
ALP (IU/L) & $90.70 \pm 27.39$ & $79.55 \pm 24.92$ & 0.00 \\
GGT (IU/L) & $41.19 \pm 33.45$ & $27.80 \pm 39.23$ & 0.00 \\
ALB (g/L) & $46.46 \pm 3.01$ & $46.19 \pm 3.01$ & 0.41 \\
TG (mmol/L) & $2.13 \pm 1.63$ & $1.41 \pm 1.12$ & 0.00 \\
CHOL (mmol/L) & $5.32 \pm 1.10$ & $5.02 \pm 0.97$ & 0.00 \\
HDL-C (mmol/L) & $1.21 \pm 0.26$ & $1.33 \pm 0.27$ & 0.00 \\
LDL-C (mmol/L) & $3.50 \pm 0.83$ & $3.14 \pm 0.83$ & 0.00 \\
Blood glucose & $5.69 \pm 1.19$ & $5.31 \pm 0.98$ & 0.00 \\
(mmol/L) & & & \\
\hline
\end{tabular}

Note: All measurement data are expressed as mean \pm standard deviation.

\section{Statistical Analysis}

All data were expressed as mean \pm standard deviation (SD). $t$-tests were used for numerical variables, and $\chi^{2}$ tests were used for categorical variables. Variables with $\mathrm{P}<0.05$ on univariate analysis entered a multiple logistic regression model. $P$ values $<0.05$ were considered statistically significant, and the statistical software used was SPSS.22. Logistic expression analysis was used to select the risk factors of NAFLD in the non-obese population.

\section{Results}

\section{Gender Distribution of NAFLD in Non-Obese and Obese Populations}

The occurrence of NAFLD was higher in the obese group than in the non-obese group $(\mathrm{P}<0.001)$, regardless of gender. The occurrence of NAFLD in female patients in the non-obese group was lower than that in male patients (male 14.25\%, female $7.5 \%, \mathrm{P}=0.001$ ), while in the obese group, no difference could be found between the genders (male 62.4\%, female $62.03 \%, \mathrm{P}=0.934$ ) (Figure 2). 


\section{(\%)}

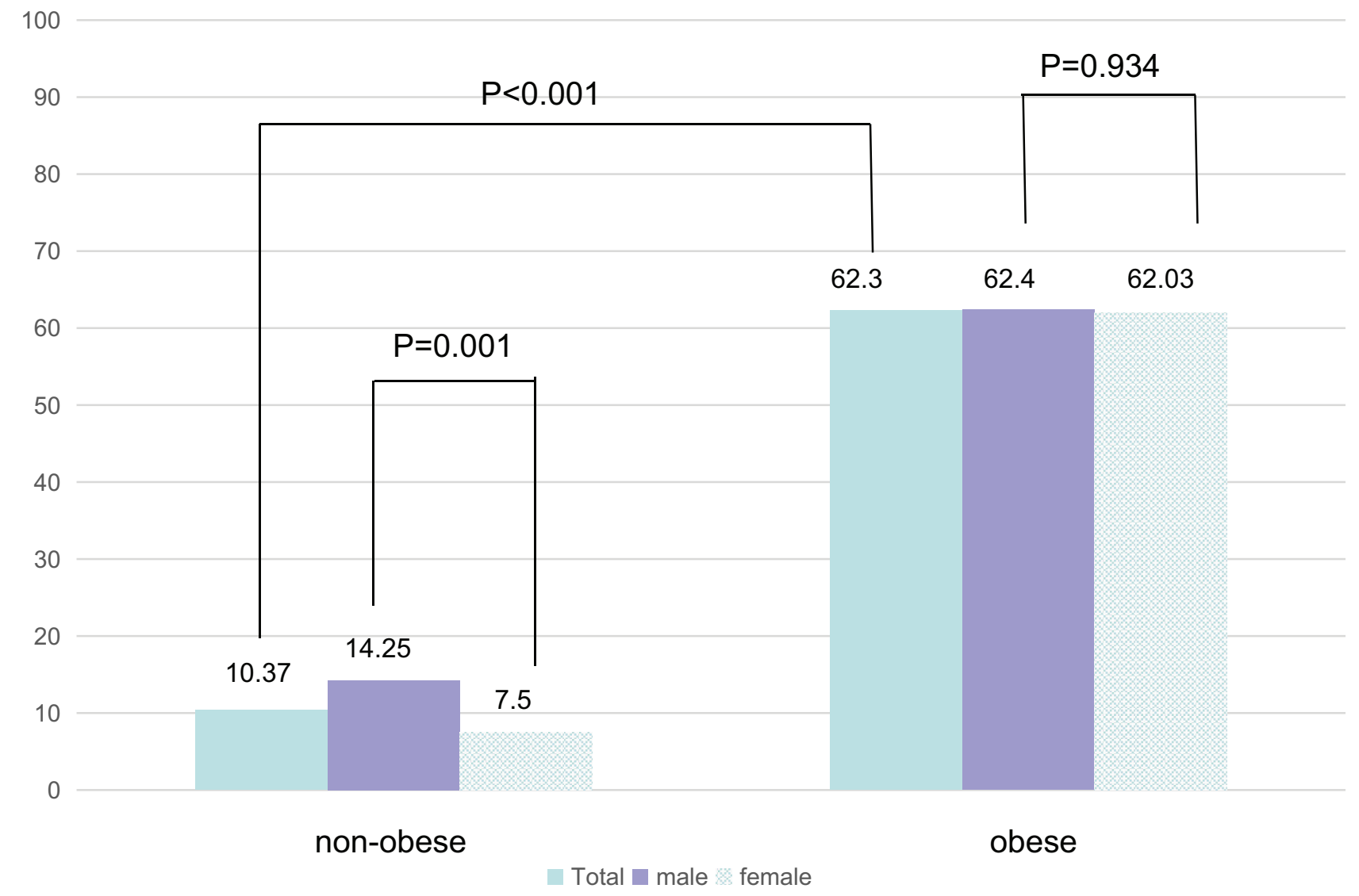

Figure 2 Gender distribution of occurrence of NAFLD in non-obese and obese population. Occurrence of NAFLD was higher in obese group than in non-obese group $(p<0.00 \mathrm{I})$. There was a statistical difference in the occurrence of NAFLD between male and female in non-obese group ( $p=0.00 \mathrm{I}$ ), no statistical difference was found in obese group $(p=0.934)$.

\section{Age Distribution of NAFLD in Non-Obese and Obese Populations}

The occurrence of NAFLD increased with age, with 50-59 years being the peak age of incidence in all groups. In patients aged 30-39 years, the proportion of NAFLD in the obese group was higher than that in the non-obese group $(P=0.005)$, while there was no difference in the incidence of NAFLD between the obese and non-obese groups with increasing age, and no difference in gender could be found (Figure 3).

In the non-obese population, the NAFLD incidence in male patients increased significantly in the age range of 40-49 years and 50-59 years, while in female patients, the incidence peaked in the age range of 50-59 years (Figure 3A).

In the obese population, the incidence of NAFLD in male patients remained basically the same across the three age groups: $30-39$ years, $40-49$ years, and 50-59 years; the incidence peaked in female patients in the age range of 50-59 years (Figure 3B).

\section{Distribution of TG Levels in Non-Obese and Obese Populations}

In order to control CHOL, LDL-C and other factors, the effect of TG on NAFLD was studied more accurately. CHOL and LDL-C of patients in each TG group were controlled within the normal range. The proportion of TG level distribution in obese and non-obese patients with NAFLD was slightly different. The proportion of elevated $\mathrm{TG}(\mathrm{TG} \geq 2.26 \mathrm{mmol} / \mathrm{L})$, marginal elevated TG $(1.7 \mathrm{mmol} /$ $\mathrm{L} \leq \mathrm{TG} \leq 2.25 \mathrm{mmol} / \mathrm{L})$, and normal $\mathrm{TG}(\mathrm{TG}<1.7 \mathrm{mmol} / \mathrm{L})$ in obese patients with NAFLD was roughly average, $40.15 \%, 22.39 \%, 37.45 \%$ respectively, while the majority of the non-obese patients with NAFLD showed normal TG 
A

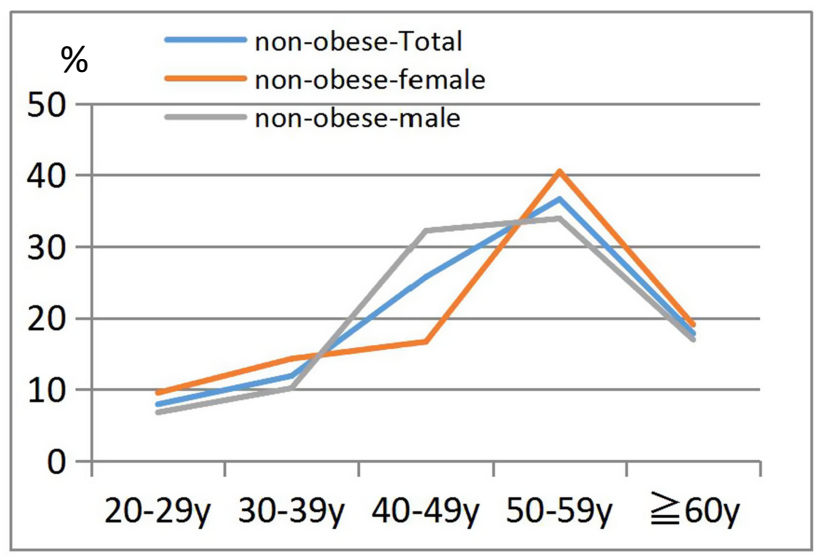

Age distribution of NAFLD in non-obese population
B

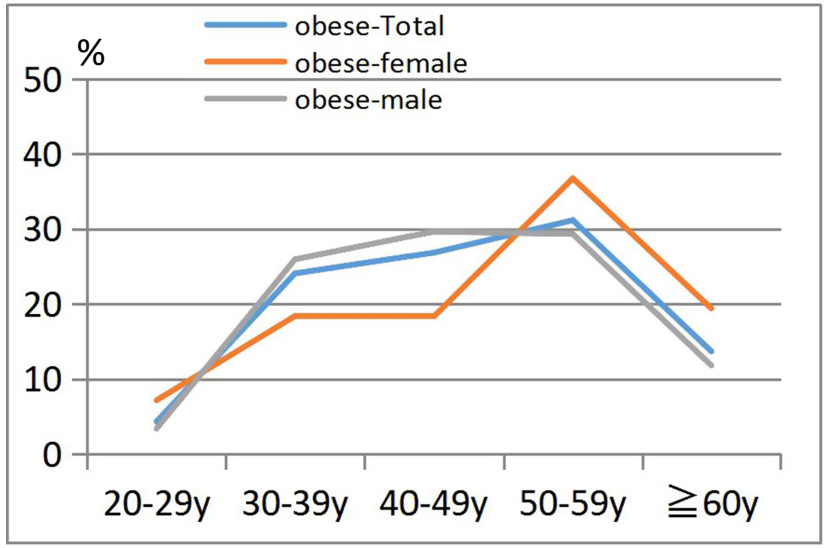

Age distribution of NAFLD in obese population

Figure 3 Age distribution of NAFLD in non-obese and obese population.

Notes: (A) In non-obese population, the NAFLD incidences in male increased significantly in the age range of 40-49y and 50-59y, while female incidence peaked in the 50-59y group. (B) In obese population, the incidence of NAFLD in male is basically the same in the three age groups of 30-39y, 40-49y and 50-59y, and the peak in female is still 50-59y group.

levels, $67.44 \%, 16.28 \%, 16.28 \%$, respectively (Figure $4 \mathrm{~A}$ and $\mathrm{B})$.

In a separate comparison between the obese and nonobese populations, there was no statistical difference in the distribution of TG levels between obese patients with NAFLD and the total obese population, while the proportions of elevated TG (16.28\%) and edge elevated TG (16.28\%) in non-obese patients with NAFLD were statistically higher than those in the total non-obese population $(5.29 \%, 7.37 \%)$ $(\mathrm{P}=0.005, \mathrm{P}=0.019)$. This indicates that elevated $\mathrm{TG}$ and edge elevated TG may be closely related to the occurrence of NAFLD in the non-obese population (Figure 4C).

\section{CHOL Levels and Occurrence of NAFLD in Non-Obese and Obese Populations}

In order to better study the effect of CHOL on NAFLD, the TG of patients in each CHOL group was controlled within the normal range. There was no statistical difference found in the distribution of CHOL levels between the obese and non-obese populations or between those with and without NAFLD (Figure 5).

\section{Constituent Ratio of Different Blood Glucose Levels in Non-Obese and Obese Populations}

In this study, blood glucose levels were stratified into 3 groups: normal blood glucose, impaired fasting glucose, and diabetes mellitus.
Among obese patients with NAFLD, no difference was found in the distribution of blood glucose levels between male and female patients, the prevalence rates of male in the normal blood glucose group, the impaired fasting glucose group and the diabetes group were $70.03 \%, 11.78 \%, 18.18 \%$, respectively, and the prevalence rates of female were $66.33 \%, 14.29 \%, 19.39 \%$, respectively (Figure 6A).

Among non-obese patients with NAFLD, the majority of female patients had normal blood glucose (92.68\%), and the constituent ratio of blood glucose levels in male patients was similar to that in obese patients with NAFLD,75\%,13.3\%,11.67\%, respectively (Figure 6B).

When all cases were grouped according to male/female, obese/non-obese, and NAFLD/non-NAFLD, the proportions of normal blood glucose were higher in the non-obese population, the non-obese NAFLD group, and non-obese female patients with NAFLD than those in the obese population, the obese NAFLD group, and obese female patients with NAFLD $(P<0.01)$; however, this pattern were not found among male patients with NAFLD $(\mathrm{p}=0.237)$. The constituent ratio of blood glucose levels in patients with NAFLD was similar to that in the total population (Figure 6C).

\section{Risk Factors for NAFLD in Non-Obese Populations}

In non-obese population, the differences of baseline characteristics between the NAFLD group (CAP $\geq 240 \mathrm{db} / \mathrm{m}$ ) and non-NAFLD group $(\mathrm{CAP}<240 \mathrm{db} / \mathrm{m})$ were analyzed 
A

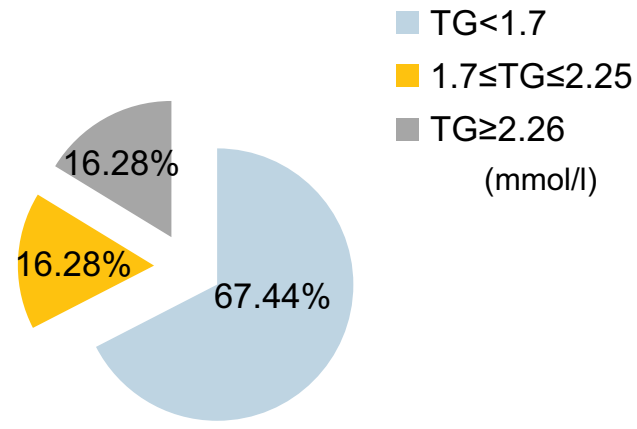

TG levels in non-obese NAFLD population
B

$\mathrm{TG}<1.7$

$1.7 \leq \mathrm{TG} \leq 2.25$

$\mathrm{TG} \geq 2.26$

$(\mathrm{mmol} / \mathrm{l})$

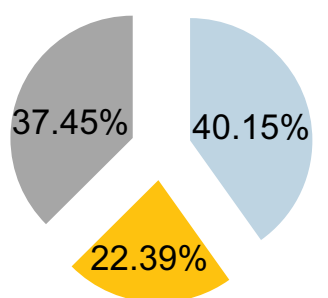

TG levels in obese NAFLD population

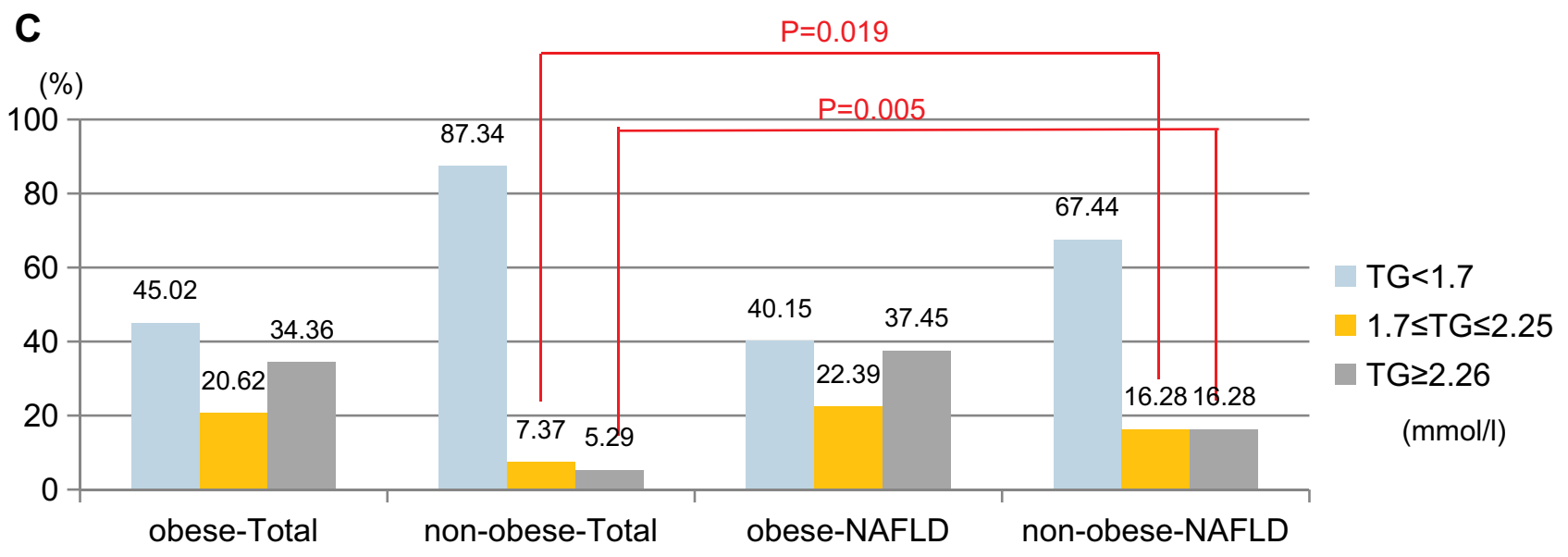

Distribution of TG levels in non-obese and obese NAFLD population

Figure 4 Distribution of TG levels in non-obese and obese population.

Notes: (A) The proportion of elevated TG, edge elevated TG and normal TG in non-obese NAFLD patients was $16.28 \%, 16.28 \%$ and $67.44 \%$, respectively, normal TG accounts for the majority. (B) The proportion of elevated TG, edge elevated TG and normal TG in obese NAFLD patients was $37.45 \%, 22.39 \%$ and $40.15 \%$, respectively. (C) The proportion of elevated TG and edge elevated TG in non-obese NAFLD patients were statistically higher than those in non-obese total population, $p=0.005$ and 0.019 , respectively.

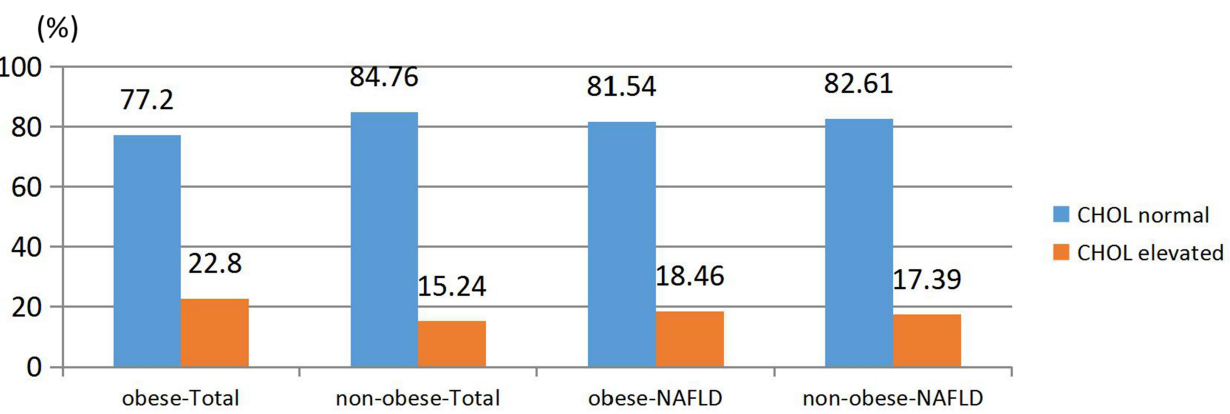

Figure $5 \mathrm{CHOL}$ levels and occurrence of NAFLD in non-obese and obese population. No statistical difference found in the distribution of CHOL levels between obese and non-obese population $(p>0.05)$.

by multivariate logistics regression analysis, the results of parameters, including age, gender, BMI, TG, CHOL and blood glucose levels, showed that $\mathrm{BMI}(\mathrm{OR}=1.311$, $\mathrm{P}=0.000)$ and $\mathrm{TG}(\mathrm{OR}=2.545, \mathrm{P}=0.000)$ were risk factors for NAFLD in the non-obese population (Table 3).

\section{Discussion}

NAFLD has become the most critical chronic liver disease in the world currently. The increase in NAFLD has become a significant public health concern because NAFLD is associated with increased mortality from liver- 

A
normal blood glucose
impaired fasting glucose ( IFG)
diabetes mellitus (DM)

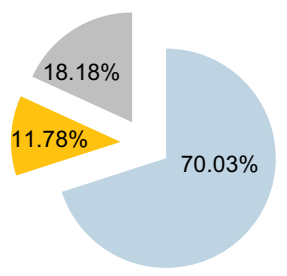

obese male-NAFLD

$$
\begin{aligned}
& \text { normal blood glucose } \\
& \text { impaired fasting glucose ( IFG) } \\
& \text { diabetes mellitus (DM) }
\end{aligned}
$$

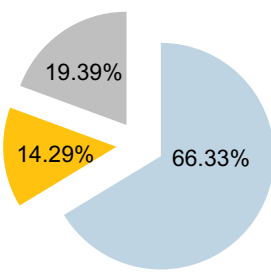

obese female-NAFLD

B

Distribution of blood glucose levels in obese male and female NAFLD patients
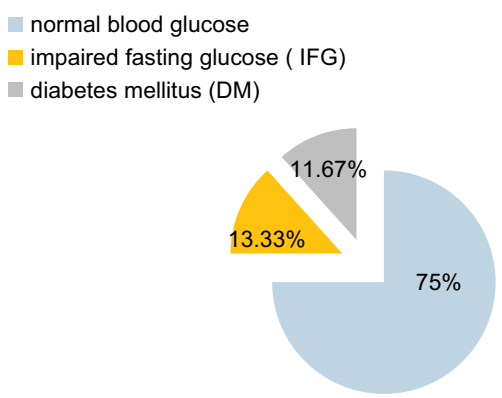

non-obese male NAFLD
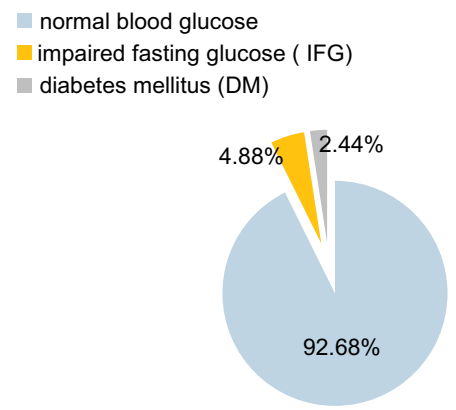

non-obese female NAFLD

C

Distribution of blood glucose levels in non-obese male and female NAFLD patients

\section{normal blood glucose impaired fasting glucose ( IFG) diabetes mellitus (DM)}

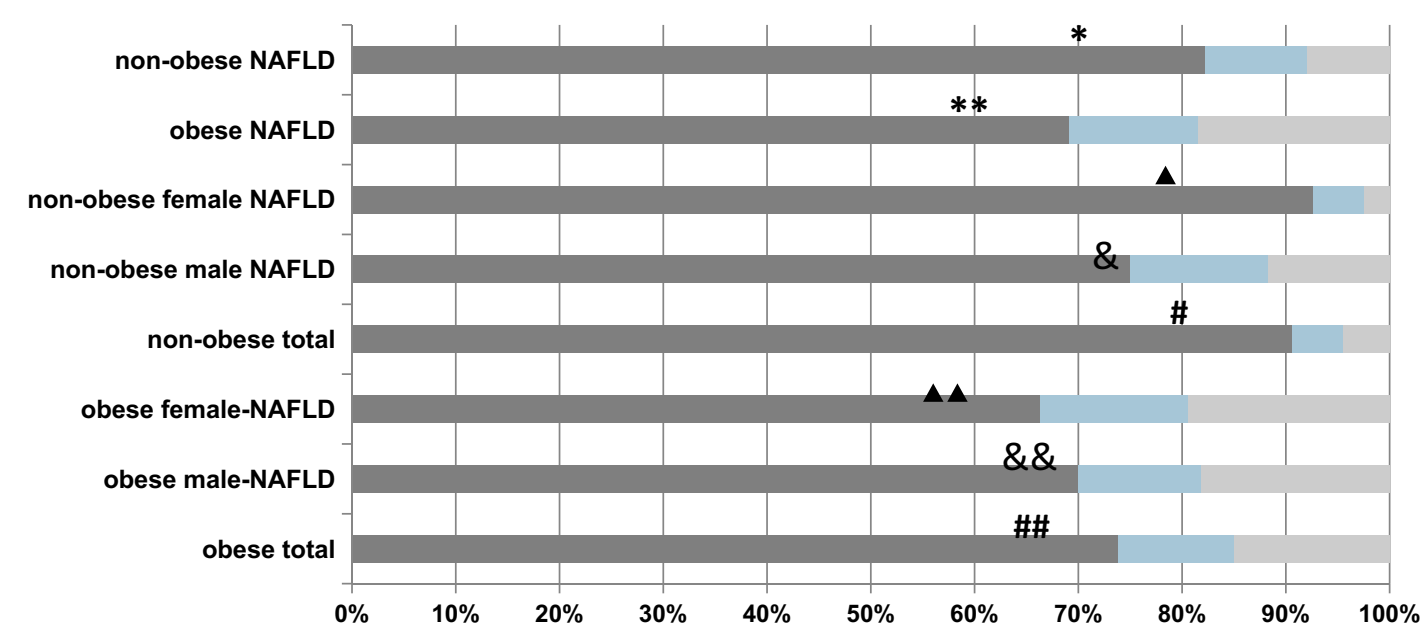

$\left({ }^{*}\right.$ s $^{* *} P<0.01, \quad \# v s \# \#<0.01, \quad \Delta v s \Delta \Delta p<0.01 \quad$ \&vs\&\& $\left.p=0.237\right)$

\section{Constituent ratio of different glucose levels in non-obese and obese population}

Figure 6 Constituent ratio of different blood glucose levels in non-obese and obese population.

Notes: (A) In obese NAFLD patients, no difference could be found in the distribution of blood glucose levels between male and female ( $p>0.05$ ). (B) In non-obese NAFLD patients, the majority of female patients have normal blood glucose, and the constituent ratio of blood glucose levels in male patients was similar to those of obese NAFLD patients ( $<<0.05$ ). (C) *proportion of normal blood glucose in non-obese NAFLD group. **proportion of normal blood glucose in obese NAFLD group. $\mathbf{\Delta}$ proportion of normal blood glucose in non-obese female NAFLD group. $\mathbf{\Delta} \boldsymbol{\Delta}$ proportion of normal blood glucose in obese female NAFLD group. ${ }^{*}$ proportion of normal blood glucose in non-obese total group. ${ }^{\text {\#\# }}$ proportion of normal blood glucose in obese total group. \& proportion of normal blood glucose in non-obese male NAFLD group. ${ }^{\text {\& }}$ proportion of normal blood glucose in obese male NAFLD group. The proportions of normal blood glucose were higher in non-obese population, non-obese NAFLD group and non-obese female NAFLD patients than those in obese population, obese NAFLD group and obese female NAFLD patients, respectively $(p<0.05)$. 
Table 3 Results of Regression Analysis of NAFLD in Non-Obese NAFLD Patients

\begin{tabular}{|l|l|l|l|}
\hline Clinical Parameters & $\mathbf{p}$ & OR & $\mathbf{9 5 \%} \mathbf{C I}$ \\
\hline Age & 0.480 & $1.00 \mathrm{I}$ & $0.998-1.004$ \\
Sex & 0.360 & 1.249 & $0.776-2.012$ \\
BMI & $0.000^{*}$ & $1.31 \mathrm{I}$ & $0.130-1.520$ \\
ALP & $0.24 \mathrm{I}$ & 1.584 & $0.734-3.416$ \\
GGT & 0.563 & 1.222 & $0.619-2.4 \mathrm{II}$ \\
TG & $0.000^{*}$ & 2.545 & $1.552-4.172$ \\
CHOL & 0.660 & 0.836 & $0.376-1.858$ \\
LDL-C & 0.675 & 1.209 & $0.498-2.93 \mathrm{I}$ \\
Blood glucose & 0.426 & 1.294 & $0.686-2.439$ \\
\hline
\end{tabular}

Note: ${ }^{*} p<0.05$.

related and liver-unrelated causes. ${ }^{10-12}$ The pathogenesis of NAFLD is not clear, and there is no effective drug for preventing the development of NAFLD to date. ${ }^{8}$ As a manifestation of the systemic metabolic syndrome, NAFLD is closely related to the metabolism of glucose and lipids and is often seen in obese populations. However, there are many non-obese populations who are diagnosed with NAFLD during clinic examinations, and the mechanism behind the occurrence of non-obese NAFLD is even less understood. ${ }^{9}$ The largest communitybased study from the East was conducted in Korea. Kwon et $\mathrm{al}^{13}$ reported the prevalence of non-obese NAFLD $(\mathrm{BMI}<25 \mathrm{~kg} / \mathrm{m} 2)$ to be $12.6 \%$ in 29,994 subjects presenting for a general medical examination. Patients with lean NAFLD are generally asymptomatic and their liver disease remains undiagnosed or incidentally detected by imaging. Compared to obese patients with NAFLD, patients with lean NAFLD have a lower body weight and waist circumference. Studies have reported that patients with lean NAFLD tend to be younger, male, have higher hemoglobin levels, lower blood pressure, fasting glucose and glycated hemoglobin (HbA1c) levels. ${ }^{14-16}$ Exploring the clinical characteristics of non-obese NAFLD may reflect how its pathogenesis is different from that of obese NAFLD.

NAFLD is defined as a sexually dimorphic disease, a characteristic that has attracted much attention in recent years. Increasing evidence shows that gender and age are related to the pathogenesis and progression of NAFLD. ${ }^{17}$ The incidence of NAFLD in postmenopausal women seems to be higher than that in premenopausal women, which shows a similar age relationship to that in male patients with NAFLD. A decrease in estrogen levels and a decline in ovarian function may be related to this phenomenon. ${ }^{18}$ Gutierrez-Grobe et al reported that the prevalence of NAFLD in premenopausal, postmenopausal, and polycystic ovarian syndrome women suggests that NAFLD is more prevalent in postmenopausal women and women with polycystic ovarian syndrome than in premenopausal women. ${ }^{19}$ Estrogen levels may have a protective effect against NAFLD in women. Studies on animal models also showed that estrogen deficiency aggravated the deterioration of non-alcoholic steatohepatitis in mice with NAFLD. ${ }^{20}$ Limited studies have also shown that hormone replacement therapy may have a beneficial effect on NAFLD. ${ }^{21}$ However, it is not clear whether these characteristics exist in non-obese patients with NAFLD. Our results showed that the occurrence of NAFLD was higher in the obese group than in the non-obese group, regardless of gender. However, in non-obese individuals, the incidence of NAFLD in female patients was significantly lower than that in male patients, suggesting that the protective effect of estrogen against NAFLD is more obvious in non-obese populations.

NAFLD incidence in different age groups also showed the possible role of estrogen. In the study of Japanese population, ${ }^{22}$ Kenichi Nishioji found that the prevalence of NAFLD peaked earlier in non-obese males than in females, and this difference may be related to the effect of hormones on body metabolism. The occurrence of NAFLD increased with age, with 50-59 years of age being the peak age of incidence in all groups. In the obese population, the incidence of NAFLD in male patients remained basically the same across the three age groups of 30-39 years, 40-49 years, and 50-59 years, while in the non-obese population, the NAFLD incidence in male patients was higher in the age groups of 40-49 years and 50-59 years. The incidence of NAFLD in female patients peaked in the age range of 50-59 years in both the obese and non-obese populations. These results show that the onset age of NAFLD is less in obese male populations. The incidence of NAFLD increasing in female patients around the age of 50-59 years may be due to the onset of menopause.

Elevated lipid and blood glucose are believed to be closely related to obesity and NAFLD, and some nonobese populations have elevated blood glucose and lipid levels that may lead to NAFLD. To prevent the interaction between different lipid components, we chose cases with normal CHOL and LDL-C levels for TG analysis. Similarly, we chose cases with normal TG and LDL-C levels for CHOL analysis. In accordance with the 
stratification standard of dyslipidemia, ${ }^{23}$ we divided the TG levels into three groups: normal TG $(\mathrm{TG}<1.7 \mathrm{mmol} /$ L), marginal elevated $\mathrm{TG}(1.7 \leq \mathrm{TG} \leq 2.25 \mathrm{mmol} / \mathrm{L})$, and elevated $\mathrm{TG}(\mathrm{TG} \geq 2.26 \mathrm{mmol} / \mathrm{L})$. The results showed that the proportion of TG level distribution in the obese and non-obese populations was different. The proportion of normal TG levels in the obese population was significantly lower than that in the non-obese population, and a similar pattern was also found in patients with NAFLD. As expected, there is a significant proportion of people with normal TG levels in non-obese patients with NAFLD. This suggests that the "elevated" and "marginal elevated" TG levels may be closely related to the occurrence of NAFLD in non-obese populations, while NAFLD can also occur in non-obese patients with normal TG levels. Therefore, nonobese populations with normal TG levels should also be monitored for NAFLD, especially those with "elevated" and "marginal elevated" TG levels. It is worth noting that no statistical difference was found either in the distribution of CHOL levels between the obese and non-obese participants or between the patients with and without NAFLD.

Elevated blood glucose level is also one of the manifestations of metabolic abnormality. Constituent ratios of different glucose levels between obese and non-obese, NAFLD and non-NAFLD, and male and female participants were analyzed. We found that, in general, the proportion of people with abnormal blood glucose in the obese population was higher than that in the non-obese population. The constituent ratio of different glucose levels in patients with NAFLD was similar to that in the total population. There was no sex-related difference in the constituent ratio of different blood glucose levels in obese patients with NAFLD. Normal blood glucose levels were found in $65 \%-70 \%$ of patients with NAFLD. In non-obese patients with NAFLD, the composition ratios of blood glucose levels in male patients were similar to those in obese patients with NAFLD, while they were significantly different in female patients, with less than $10 \%$ of nonobese females showing abnormal blood glucose levels. This suggests that female patients with normal blood glucose should be monitored for NAFLD more closely than male patients.

Finally, we analyzed the risk factors for NAFLD in the non-obese population. The results showed that BMI and TG were still risk factors for NAFLD in the non-obese population. Most studies believe that there is a close correlation between NAFLD and BMI. ${ }^{30}$ The higher the BMI, the higher the incidence of metabolic syndrome and the greater the probability of NAFLD, high BMI is an independent risk factor for NAFLD. Our results showed that BMI was still a risk factor for NAFLD in non-obese patients even though their BMI $<25 \mathrm{~kg} / \mathrm{m}^{2}$. These findings remind us that reasonable dietary and physical activity status should also be advocated in people with normal BMI, which is helpful to prevent the development of NAFLD. In addition, high-fat diet is closely related to fatty liver, normal hepatocytes contain about $4 \%-7 \%$ of lipids, of which TG accounts for about $1 / 2$. Fatty liver can be caused when the content of TG in the liver increases. Our data showed that the average level of TG in NAFLD group was higher than that of non-NAFLD in non-obese population. It's very interesting that we found the "edge elevated“ TG level $(1.7 \mathrm{mmol} / \mathrm{L} \leq \mathrm{TG} \leq 2.25 \mathrm{mmol} / \mathrm{L})$ may be closely related to the occurrence of NAFLD in nonobese population. Results above remind us that attention should also be paid to monitor NAFLD in those people with normal TG and BMI in favor of early intervention. Certainly, genetic and other factors may also be related to the occurrence of NAFLD in non-obese people.

In summary, compared with obese population, the incidence of NAFLD in non-obese population was relatively low and more frequently in male than in female, the peak age of NAFLD occurrence in non-obese male patients was also delayed. BMI and TG should still be controlled to avoid the occurrence of NAFLD although the BMI of such patients is normal. Due to the normal BMI, non-obese NAFLD may be ignored by patients and can result in potential liver lesions and disease progression; therefore, more attention should be paid. However, BMI fails to address body fat distribution. ${ }^{24}$ Visceral abdominal adiposity, which is associated with the metabolic syndrome, is thought to be a key link to NAFLD, it plays a far more important role than subcutaneous fat in the pathogenesis of insulin resistance. ${ }^{25-27}$ We expected the study of the relationship between visceral obesity and non-obese NAFLD patients. More importantly, there is no dispute that NAFLD is closely related to several endocrine factors (hormone, glycolipid metabolism), and the concept of "endocrine NAFLD" has been once proposed, ${ }^{28,29}$ which may be a new direction for future research on the mechanism and treatment of NAFLD.

\section{Statement of Ethics}

The research was conducted ethically in accordance with the World Medical Association Declaration of Helsinki 
and was approved by the Ethics Committee of ChinaJapan Union Hospital, Jilin University (2016ks009). Participation was voluntary and all respondents completed informed consent.

\section{Disclosure}

The authors declare that they have no competing interests.

\section{References}

1. Wattacheril J. Extrahepatic Manifestations of Nonalcoholic Fatty Liver Disease. Gastroenterol Clin North Am. 2020;49(1):141-149. doi:10.1016/j.gtc.2019.10.002

2. Wang AY, Dhaliwal J, Mouzaki M. Lean non-alcoholic fatty liver disease. Clin Nutr. 2019;38(3):975-981. doi:10.1016/j.clnu.2018.08. 008

3. Tu LN, Showalter MR, Cajka T, et al. Metabolomic characteristics of cholesterol-induced non-obese nonalcoholic fatty liver disease in mice. Sci Rep. 2017;7(1):6120. doi:10.1038/s41598-017-05040-6

4. Ahadi M, Molooghi K, Masoudifar N, Namdar AB, Vossoughinia H, Farzanehfar M. A review of non-alcoholic fatty liver disease in non-obese and lean individuals [published online ahead of print, 2020 Nov 20]. J Gastroenterol Hepatol. 2020. doi:10.1111/jgh.15353

5. Schiffer L, Kempegowda P, Arlt W, O'Reilly MW. MECHANISMS IN ENDOCRINOLOGY: the sexually dimorphic role of androgens in human metabolic disease. Eur J Endocrinol. 2017;177(3):R125R143. doi:10.1530/EJE-17-0124

6. WHO Expert Consultation. Appropriate body-mass index for Asian populations and its implications for policy and intervention strategies [published correction appears in Lancet]. Lancet. 2004;363 (9403):157-163. doi:10.1016/S0140-6736(03)15268-3

7. Jian-gao F; Chinese Liver Disease Association. Guidelines for management of nonalcoholic fatty liver disease: an updated and revised edition. Zhonghua Gan Zang Bing Za Zhi. 2010;18(3):163-166.

8. Friedman SL, Neuschwander-Tetri BA, Rinella M, Sanyal AJ. Mechanisms of NAFLD development and therapeutic strategies. Nat Med. 2018;24(7):908-922. doi:10.1038/s41591-018-0104-9

9. Liu CJ. Prevalence and risk factors for non-alcoholic fatty liver disease in Asian people who are not obese. J Gastroenterol Hepatol. 2012;27(10):1555-1560. doi:10.1111/j.1440-1746.2012.072 22.x

10. Adams LA, Lymp JF, St Sauver J, et al. The natural history of nonalcoholic fatty liver disease: a population-based cohort study. Gastroenterology. 2005;129(1):113-121. doi:10.1053/j.gastro.2005. 04.014

11. Ekstedt M, Franzén LE, Mathiesen UL, et al. Long-term follow-up of patients with NAFLD and elevated liver enzymes. Hepatology. 2006;44(4):865-873. doi:10.1002/hep.21327

12. Targher G, Byrne CD, Tilg H. NAFLD and increased risk of cardiovascular disease: clinical associations, pathophysiological mechanisms and pharmacological implications. Gut. 2020;69(9):1691-1705. doi:10.1136/gutjnl-2020-320622

13. Kwon YM, Oh SW, Hwang SS, Lee C, Kwon H, Chung GE. Association of nonalcoholic fatty liver disease with components of metabolic syndrome according to body mass index in Korean adults. Am J Gastroenterol. 2012;107(12):1852-1858. doi:10.1038/ajg.20 12.314

14. Younossi ZM, Stepanova M, Negro F, et al. Nonalcoholic fatty liver disease in lean individuals in the United States. Medicine. 2012;91 (6):319-327. doi:10.1097/MD.0b013e3182779d49
15. Akyuz U, Yesil A, Yilmaz Y. Characterization of lean patients with nonalcoholic fatty liver disease: potential role of high hemoglobin levels. Scand J Gastroenterol. 2015;50(3):341-346. doi:10.3109/ 00365521.2014 .983160

16. Vos B, Moreno C, Nagy N, et al. Lean non-alcoholic fatty liver disease (Lean-NAFLD): a major cause of cryptogenic liver disease. Acta Gastroenterol Belg. 2011;74(3):389-394.

17. Lee JY, Shin DW, Oh JW, et al. Non-alcoholic fatty liver disease as a risk factor for female sexual dysfunction in premenopausal women. PLoS One. 2017;12(8):e0182708. doi:10.1371/journal.pone.0182708

18. Ballestri S, Nascimbeni F, Baldelli E, Marrazzo A, Romagnoli D, Lonardo A. NAFLD as a sexual dimorphic disease: role of gender and reproductive status in the development and progression of nonalcoholic fatty liver disease and inherent cardiovascular risk. $A d v$ Ther. 2017;34(6):1291-1326. doi:10.1007/s12325-017-0556-1

19. Gutierrez-Grobe Y, Ponciano-Rodríguez G, Ramos MH, Uribe M, Méndez-Sánchez N. Prevalence of non alcoholic fatty liver disease in premenopausal, postmenopausal and polycystic ovary syndrome women. The role of estrogens. Ann Hepatol. 2010;9(4):402-409. doi:10.1016/S1665-2681(19)31616-3

20. Matsushita N, Hassanein MT, Martinez-Clemente M, et al. Gender difference in NASH susceptibility: roles of hepatocyte $\operatorname{Ikk} \beta$ and Sult1e1. PLoS One. 2017;12(8):e0181052. doi:10.1371/journal.pone. 0181052

21. Venetsanaki V, Polyzos SA. Menopause and non-alcoholic fatty liver disease: a review focusing on therapeutic perspectives. Curr Vasc Pharmacol. 2019;17(6):546-555. doi:10.2174/157016111666618071 1121949

22. Nishioji K, Sumida Y, Kamaguchi M, et al. Prevalence of and risk factors for non-alcoholic fatty liver disease in a non-obese Japanese population, 2011-2012. J Gastroenterol. 2015;50(1):95-108. doi:10. 1007/s00535-014-0948-9

23. Joint committee for guideline revision. 2016 Chinese guidelines for the management of dyslipidemia in adults. $J$ Geriatr Cardiol. 2018;15(1):1-29. doi:10.11909/j.issn.1671-5411.2018.01.011

24. Cusi K. Role of obesity and lipotoxicity in the development of nonalcoholic steatohepatitis: pathophysiology and clinical implications. Gastroenterology. 2012;142(4):711-725.e6. doi:10.10 53/j.gastro.2012.02.003

25. Yim JY, Kim D, Lim SH, et al. Sagittal abdominal diameter is a strong anthropometric measure of visceral adipose tissue in the Asian general population. Diabetes Care. 2010;33(12):2665-2670. doi: $10.2337 / \mathrm{dc} 10-0606$

26. Kuk JL, Lee S, Heymsfield SB, Ross R. Waist circumference and abdominal adipose tissue distribution: influence of age and sex. $\mathrm{Am}$ J Clin Nutr. 2005;81(6):1330-1334. doi:10.1093/ajen/81.6.1330

27. McLaughlin T, Lamendola C, Liu A, Abbasi F. Preferential fat deposition in subcutaneous versus visceral depots is associated with insulin sensitivity. J Clin Endocrinol Metab. 2011;96(11):E1756E1760. doi:10.1210/jc.2011-0615

28. Lonardo A, Mantovani A, Lugari S, Targher G. NAFLD in some common endocrine diseases: prevalence, pathophysiology, and principles of diagnosis and management. Int J Mol Sci. 2019;20 (11):2841. doi:10.3390/ijms20112841

29. Lonardo A, Carani C, Carulli N, Loria P. 'Endocrine NAFLD' a hormonocentric perspective of nonalcoholic fatty liver disease pathogenesis. J Hepatol. 2006;44(6):1196-1207. doi:10.1016/j. jhep.2006.03.005

30. Naderian M, Kolahdoozan S, Sharifi AS, et al. Assessment of Lean patients with non-alcoholic fatty liver disease in a middle income country; prevalence and its association with metabolic disorders: a cross-sectional study. Arch Iran Med. 2017;20(4):211-217. 


\section{Publish your work in this journal}

Diabetes, Metabolic Syndrome and Obesity: Targets and Therapy is an international, peer-reviewed open-access journal committed to the rapid publication of the latest laboratory and clinical findings in the fields of diabetes, metabolic syndrome and obesity research. Original research, review, case reports, hypothesis formation, expert opinion and commentaries are all considered for publication. The manuscript management system is completely online and includes a very quick and fair peer-review system, which is all easy to use. Visit http://www.dovepress.com/testimonials.php to read real quotes from published authors. 